\title{
INFORMACION
}

\section{Alteraciones bioquímicas de los lípidos en los alimentos vegetales. II. Metabolismo de los hidroperóxidos lipídicos.}

\author{
Por R. Zamora. F. J. Hidalgo y M. Alaiz \\ Instituto de la Grasa y sus Derivados. Avda. Padre García Tejero, 4 - 41012 Sevilla.
}

\section{RESUMEN}

Alteraciones bioquímicas de los lípidos en los alimentos vegetales. II. Metabolismo de los hidroperóxidos lipídicos.

Los enzimas responsables de la degradación enzimática de los lípidos en los alimentos vegetales son examinados en este trabajo. Esta parte describe la descomposición o transformación de los hidroperóxidos lipídicos producida por la lipoxigenasa, hidroperóxido liasa, enal isomerasa, alcohol deshidrogenasa, aldehído deshidrogenasa, hidroperóxido dehidrasa, óxido de aleno ciclasa y ácido 12 oxofitodienoico reductasa, así como los distintos mecanismos de control de la ruta.

PALABRAS-CLAVE: Alimento vegetal - Información (artículo) Lipoxigenasa (ruta) - Oxidación lip/dica.

\section{SUMMARY}

Biochemical changes of lipids in plant foods and feeds. II. Lipid hydroperoxides metabolism.

The enzymes of the lipoxygenase pathway in plant foods are reviewed. This part analyzes the enzymes related to the descomposition or transformation of the lipidic hydroperoxides. Lipoxygenase, hydroperoxide lyase, enal isomerase, alcohol dehydrogenase, aldehyde dehydrogenase, hydroperoxide dehydrase, alene oxide ciclase and 12-0xo-phytodienoic acid reductase in plants are described. Posible control mechanisms of the lipoxygenase pathway are also considered.

KEY-WORDS: Information (paper) - Lipid oxidation - Lipoxygenase (pathway) - Plant food.

\section{INTRODUCCION}

La parte primera de esta revisión (1) describía los enzimas que intervienen en la formación de los hidroperóxidos lipídicos en alimentos, siendo la lipoxigenasa el enzima clave de todo el proceso. Una vez formados estos hidroperóxidos, los mismos son metabolizados por todo un conjunto de enzimas que van a ser el objeto de esta segunda parte. Estos hidroperóxidos pueden ser también descompuestos mediante una serie de reacciones no enzimáticas, que no serán descritas en este trabajo, y que han sido objeto de recientes revisiones (2-4).

\section{LIPOXIGENASA}

Aunque los hidroperóxidos formados por acción de la lipoxigenasa son los sustratos de diversos enzimas que serán descritos posteriormente, se ha observado que a menudo la propia lipoxigenasa también puede "catalizar" la degradación de los mismos hidroperóxidos, creyéndose que esta propiedad se debe a la naturaleza redox del hierro en el sitio activo. La forma ferrosa del enzima puede causar una rotura homolítica del hidroperóxido generando un radical alcoxi que espontáneamente es transformado en productos secundarios. Este área de investigación es una de las más extensamente estudiadas hoy día aunque todavía no es totalmente comprendida y muchos de los resultados encontrados son a veces contradictorios.

Los distintos productos formados en estas reacciones van a depender de sí la misma se lleva a cabo en condiciones aeróbicas o anaeróbicas. En la reacción aeróbica se observa la formación de trihidroxi, oxohidroxi, hidroxi, epoxi, hidroxidién y oxodién derivados. La formación aeróbica de oxodienos fue primeramente descrita por Vioque y Holman (5), usando un extracto crudo de lipoxigenasa y posteriormente Axelrod (6) ha obtenido similares resultados con la isoenzima 3 de la lipoxigenasa de soja.

Se conoce muy poco sobre la influencia que estos compuestos tienen en la calidad de los alimentos. Se sabe que los ácidos hidroxi- y trihidroxi-octadecenoicos inducen sabor amargo y varios autores les han atribuido el sabor amargo de los cereales y leguminosas $(6,8)$. Por la misma razón se le han atribuido 
igualmente el sabor amargo de la cerveza (9). Asímismo son indirectamente responsables del flavor a viejo de esta misma bebida, que ha sido atribuido al E-2nonenal, por ser este último compuesto fácilmente generado a partir de los trihidroxi por calentamiento en condiciones ácidas. Los trihidroxi encontrados en la cerveza son análogos a los obtenidos enzimáticamente a partir del ácido linolénico y harina de cebada, por lo que estos compuestos proceden probablemente de la cebada usada en la manufactura de la misma (9).

Por lo que respecta a los epoxiderivados de los ácidos grasos se sabe que son citotóxicos y mutagénicos (10) aunque su importancia biológica o alimentaria no ha sido evaluada por ahora.

En cuanto a las reacciones anaeróbicas de la lipoxigenasa se sabe que dan lugar a mezclas de productos entre los que se encuentran pentano, oxoácidos, epóxidos y productos diméricos. Estos estudios han sido realizados principalmente con lipoxigenasa de soja $(11,12)$. Se ha comprobado que el sustrato de estas reacciones es el 13-hidroperóxido y se necesita la presencia del ácido linoleico. Garssen (11) demostró que el oxoderivado provenía del hidroperóxido y no del ácido linoleico. Cuando la concentración de lipoxigenasa es muy elevada tanto el 9- como el 13-hidroperóxido son degradados anaeróbicamente en ausencia del ácido linoleico (13). No obstante, la presencia de este ácido estimula la degradación del 13-hidroperóxido unas 1500 veces mientras que no afecta la degradación del 9-. Presumiblemente esto puede ser debido a que el ácido linoleico actúa como un donador de electrones al hierro del sitio activo. En ausencia de ácido linoleico debe operar otro donador de electrones, quizás por un mecanismo análogo al propuesto por Verhagen et al. (13) donde es el propio hidroperóxido el que dona los electrones produciendo radicales hidroxi e hidropero$x i$.

Aparte de la degradación de los hidroperóxidos aeróbica o anaeróbicamente, la lipoxigenasa puede provocar la oxidación de otros compuestos por un proceso llamado cooxidación. Ya en 1928 se constató que la harina de soja emblanquecía los carotenoides de la harina de trigo (14). Este fenómeno de oxidación de carotenos era paralelo a la oxidación de los ácidos grasos y tras la purificación de las isoenzimas de la lipoxigenasa se pudo comprobar que era ésta y no otro factor la responsable de la reacción.

La cooxidación requiere la presencia de ambos sustratos, es decir ácido graso y caroteno, y la lipoxigenasa, y esta última no puede ser reemplazada por el hidroperóxido. Hoy en día se conocen evidencias que sugieren que la lipoxigenasa media la cooxidación mediante un proceso de radicales libres (15).

Esta cooxidación de la lipoxigenasa también puede tener lugar sobre la clorofila provocando su emblan- quecimiento en un proceso cuyas repercusiones sobre la calidad de los alimentos aún está por estudiar (16).

A pesar de la evidente importancia de todas las reacciones anteriormente descritas, la producción de volátiles como consecuencia de la cascada de los ácidos linoleico y linolénico es el campo que ha sido más ampliamente estudiado por su incidencia en el flavor de determinados alimentos ya sean consumidos posteriormente en estado natural o después de su procesado. Los hidroperóxidos producidos por la lipoxigenasa pueden ser asímismo degradados por este enzima, produciendo aldehídos volátiles y otros derivados que contribuyen de una manera muy importante al flavor característico de muchas plantas y frutos.

La degradación del ácido linoleico por la lipoxigenasa $(17,18)$ produce principalmente hexanal, 2,4decadienal, 2-heptenal, 2-octenal, pentanal y 2,4nonadienal. Estos mismos volátiles se obtienen siempre independientemente de la pureza del enzima empleado en su formación aunque las proporciones relativas sí dependen mucho de aquel. La lipoxigenasa 1 de soja da principalmente hexanal mientras que la 2 y 3 dan hexanal en una concentración similar a otros aldehídos: (E)-2, (E)-4-decadienal, (E)-2,(Z)-4decadienal, (E)-2-heptenal, (E)-2-octenal, (E)-2,(E)-4nonadienal, heptanal, pentilfurano y pentanal (19).

Cuando la oxidación se lleva a cabo sobre el ácido linolénico el conjunto de compuestos volátiles difiere bastante del obtenido con el ácido linoleico. Un extracto crudo de guisantes produjo (E)-2,(Z)-4-heptadienal, propanal, 2-pentenal, acetaldehído, crotonaldehído y 2-hexenal (17). La lipoxigenasa 1 de soja pura dio por su parte solamente (E)-2,(Z)-4-hexadienal, (E)-2,(Z)4-heptadienal, (E)-2-pentenal, (E)-2-hexenal, 4,6-nonadienal, 3,5-octadién-2-ona y otros aldehídos minoritarios (20).

El tipo de volátiles producidos y la proporción relativa entre los mismos depende no sólo del ácido de partida sino también de la procedencia de la lipoxigenasa utilizada (21). Esto parece que es debido a la distinta especificidad del enzima.

Muchos trabajos han indicado que la lipoxigenasa cataliza la formación de estos productos mediante la destrucción de los hidroperóxidos via una catálisis seudoenzimática. La reacción parece ser que transcurre por una via radicalaria como lo demuestra la cooxidación del ácido oleico a octanal y nonanal durante la cooxidación del ácido linoleico por la lipoxigenasa de guisante (17). Se cree que el hierro presente en el sitio activo del enzima es el responsable de estas reacciones, análogamente a la catálisis del hierro o hemoproteínas que pueden producir volátiles a partir del hidroperóxido (21). Supuestamente la lipoxigenasa opera en un ciclo redox propuesto por Vliengenthart et al. (22) y la formación de volátiles vendría dada por la descomposición del radical alcoxi formado en la rotura del hidroperóxido. 


\section{HIDROPEROXIDO LIASA}

Independientemente de la capacidad anteriormente descrita que tiene la lipoxigenasa de transformar sus productos de oxidación, diversos autores han puesto de manifiesto la existencia de una actividad hidroperóxido liasa que es capaz de romper los productos de oxidación de los ácidos linoleico y linolénico dando lugar a compuestos aldehídicos. Como se recoge en la Figura 1 el 13-hidroperóxido de los ácidos linoleico y linolénico es convertido en el co-

\section{3-hidroperóxidos}

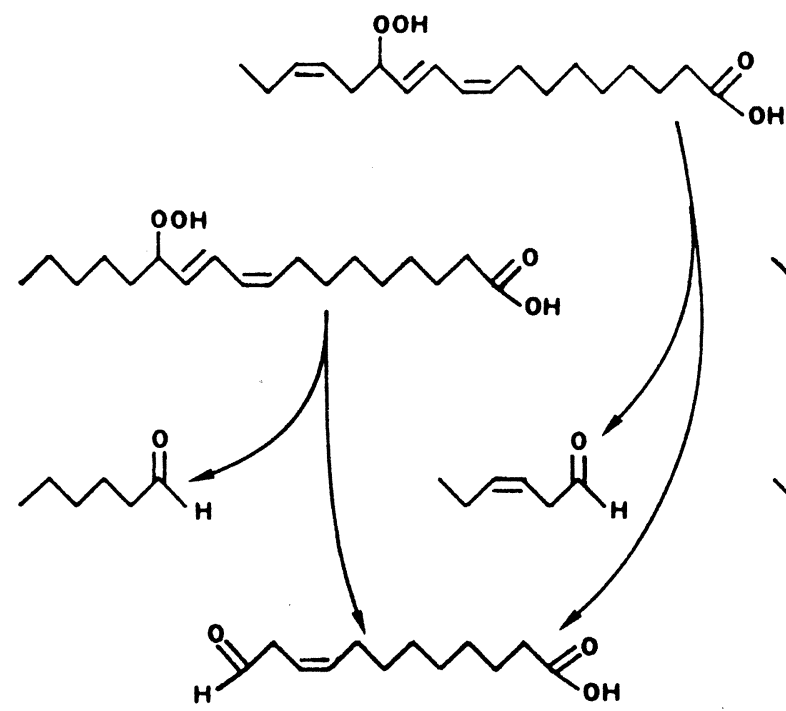

rrespondiente aldehído de 6 átomos de carbono (hexanal o (Z)-3-hexenal, a partir del 13-hidroperóxido de los ácidos linoleico y linolénico, respectivamente); y 12-oxo-(Z)-9-dodecenoico. De los 9-hidroperóxidos de los ácidos linoleico o linolénico se produce el ácido 9-oxononenoico y un aldehído C-9 (el (Z)-3nonenal o el (Z)-3,(Z)-6-nonadienal, respectivamente). De estos productos de la liasa, los aldehídos $B, \gamma-$ insaturados se isomerizan fácilmente a aldehídos $\alpha, \beta$ insaturados bien enzimáticamente o autocatalíticamente.

\section{9-hidroperóxidos}

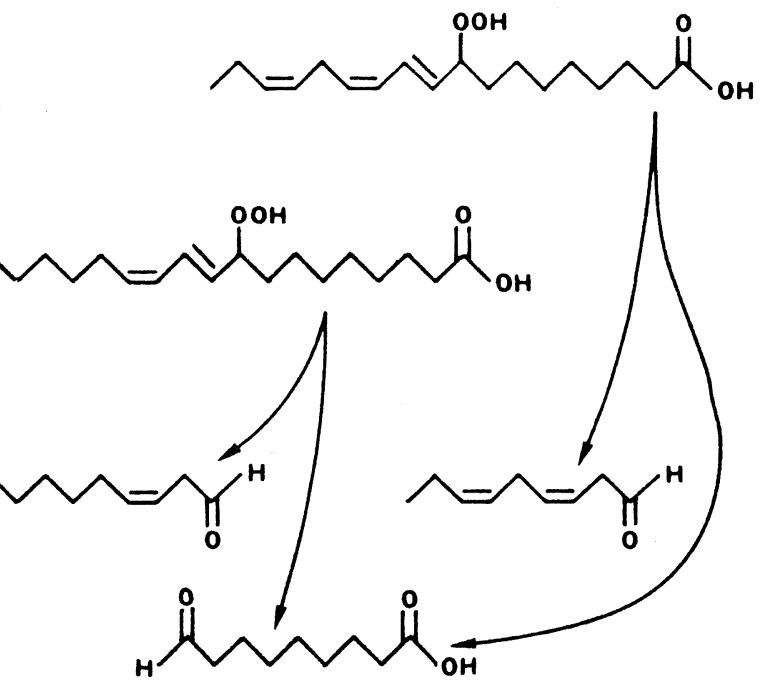

Figura 1

Degradación de los 9- y 13-hidroperóxidos de los ácidos linoleico y linolénico producida por la hidroperóxido liasa.

Aunque la hidroperóxido liasa fue encontrada primero en tejidos no verdes, posteriormente ha sido encontrada también en los tejidos de plantas verdes. La mayoría de los estudios indican que el enzima se encuentra enlazado a membranas. En tejidos verdes el enzima está localizado en los cloroplastos, o más concretamente en las "lamelas" del cloroplasto (23), $y$ en los tejidos no verdes se ha encontrado en el retículo endoplásmico, el aparato de Golgi y la membrana plasmática (24).

En la mayoría de las plantas estudiadas hasta ahora, el 13-hidroperóxido de los ácidos linoleico y linolénico es el sustrato más activo para la hidroperóxido liasa. En otras plantas, como por ejemplo el pepino, el enzima puede utilizar cualquiera de los dos isómeros, el 9- o el 13-, como sustratos (25). En el caso de la pera sólo el 9-hidroperóxido es el sustrato (26). Curiosamente los chamiñones tienen una extraña hidroperóxido liasa que utiliza como sustrato sólamente el 10(S)-hidroperóxido del ácido linoleico produciendo fragmentos de 8 y 10 átomos de carbono que son el 1-octen-3-ol y el ácido 10-oxo-(E)-8-dece- noico (Figura 2). El alcohol obtenido, 1-octen-3-ol, es el responsable del flavor característico de los champiñones (27). Es de suponer que los champiñones<smiles>CCCCC/C=C/CC(/C=C/CCCCCCC(=O)O)OO</smiles><smiles>C=CC(O)CCCCC</smiles>

Figura 2

Degradación del 10-hidroperóxido del ácido linoleico producida por la hidroperóxido liasa presente en el champiñón. 
deben poseer una lipoxigenasa extraña que cataliza la incorporación de oxígeno en el carbono 10 del ácido linoleico.

El mecanismo de acción de la hidroperóxido liasa no ha sido estudiado en profundidad hasta muy recientemente. En hojas de té, la hidroperóxido liasa tiene un requerimiento específico para la configuración $S$ del hidróperóxido como sustrato. Los $\alpha$-cetoles obtenidos por acción de la hidroperóxido dehidrasa que comentaremos posteriormente, podrían parecer a priori buenos intermediarios. Sin embargo, todos los intentos de demostrar esta hipótesis han fallado. Hoy en día se supone que la acción de la hidroperóxido liasa es similar a la rotura del hidroperóxido producida por un ácido fuerte de Lewis en un disolvente aprótico. Experimentos con oxígeno marcado apoyan esta hipótesis ya que el grupo oxo del ácido 12-oxododecenoico se originó del oxígeno molecular mientras que el grupo oxo del aldehído se originó del agua. Experimentos similares llevados a cabo con la hidroperóxido liasa anómala de los champiñones que comentábamos anteriormente han mostrado que un oxígeno del 10-hidroperóxido del ácido linoleico se convierte en el grupo hidroxilo del 1-octen-3-ol y el otro probablemente permanece como grupo oxo del ácido 10-oxo-(E)-8-decenoico (27).

\section{3-hidroperóxidos}

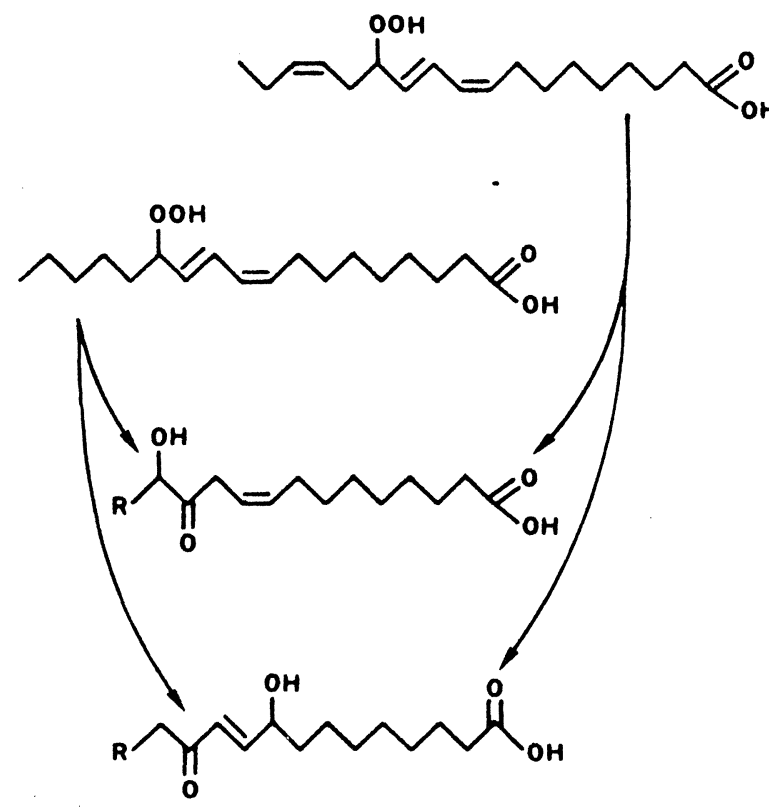

\section{ENAL ISOMERASA, ALCOHOL DESHIDROGE- NASA Y ALDEHIDO DESHIDROGENASA.}

Los productos de la hidroperóxido liasa pueden ser posteriormente transformados por una enal isomerasa. Las isomerizaciones alrededor del doble enlace son mediadas por una enal isomerasa que se cree que es común a la mayoría de las plantas (28). Phillips et al. (29) probaron la naturaleza enzimática de este proceso purificando el enzima; no obstante, isomerizaciones no enzimáticas similares es posible que ocurran en un amplio número de plantas (28).

Los aldehídos producidos por la lipoxigenasa e hidroperóxido liasa pueden ser posteriormente también reducidos a alcoholes u oxidados a ácidos por acción de la alcohol deshidrogenasa o la aldehído deshidrogenasa, respectivamente. Estas reacciones son importantes en la reducción de los niveles de aldehídos indeseables y los olores asociados a, por ejemplo, la harina de soja y en la formación de ésteres que son importantes compuestos flavor en algunos frutos.

Los aldehídos pueden ser reducidos a alcoholes por acción de la alcohol deshidrogenasa en presencia de NADH u oxidados a ácidos por medio de la aldehído - deshidrogenasa en presencia de $\mathrm{NAD}^{+}(30)$.

\section{9-hidroperóxidos}
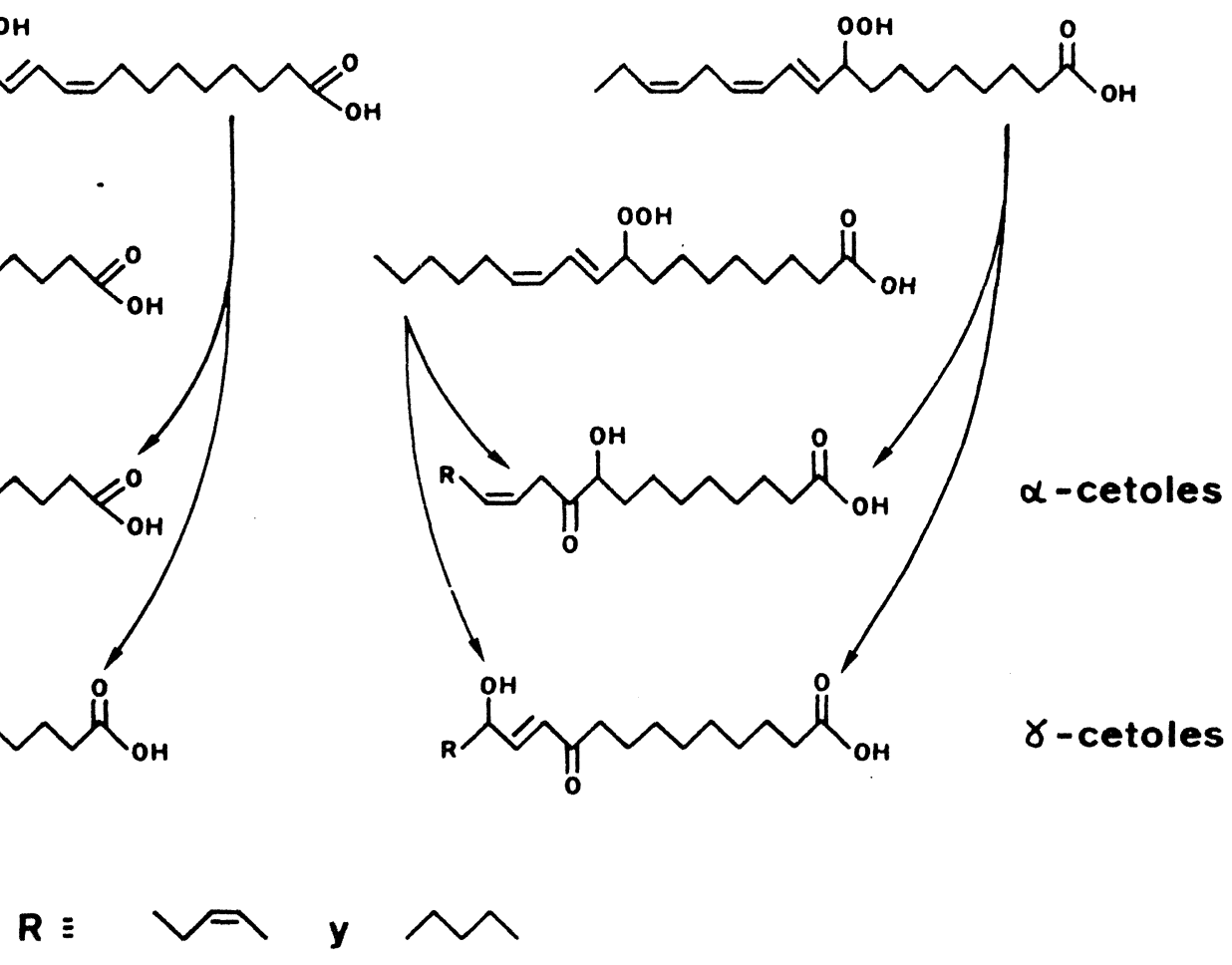

Figura 3

Formación de $\alpha$ - y $\gamma$-cetoles a partir de los hidroperóxidos de los ácidos linoleico y linolénico 


\section{HIDROPEROXIDO DEHIDRASA}

Otra de las transformaciones que pueden sufrir los hidroperóxidos es la isomerización a $\alpha$ - y $\gamma$-cetoles que se recoge en la Figura 3 (31). Esta reacción fue descubierta por Zimmerman en 1966 en lino (32) $y$ posteriormente ha sido encontrada en otras especies vegetales como cebada $(33,34)$, maíz (35), algodón (36), berenjena (37), lechuga (38), avena, (38), espinacas (38), girasol (38) y trigo (38). Estos $\alpha$ - y $\gamma$-cetoles pueden ser producidos tanto a partir del 9como del 13-hidroperóxido y durante mucho tiempo han sido considerados productos de una hipotética hidroperóxido isomerasa (31). Sin embargo, Hamberg (39) recientemente caracterizó un intermediario en la conversión enzimática del 13-hidroperóxido del ácido linoleico en $\alpha$-cetol. Este compuesto, el ácido 12,13(S)-epoxi-9(Z),11-octadecadienoico tiene una vida media de aproximadamente $33 \mathrm{~s}$ a $0^{\circ} \mathrm{C}$ y se forma por la pérdida, catalizada por un enzima, de una molécula de agua del hidroperóxido. El enzima responsable de este paso ha sido denominado hidroperóxido dehidrasa $(39,40)$.

Su mecanismo de acción ha sido estudiado y el óxido de aleno formado, a pesar de ser muy inestable, ha sido bien caracterizado (41). Así cuando se partió de hidroperóxidos marcados con ${ }^{18} \mathrm{O}$ en los oxígenos del grupo hidroperóxido y ${ }^{2} \mathrm{H}$ en las posiciones $9,10,12$ y 13, y el óxido de aleno formado se atrapó con metanol, se encontró que el producto for- mado (Figura 4) había retenido uno de los oxígenos del hidroperóxido y los deuterios de las posiciones 9 , 10 y 13 (39). Esto confirma la formación del óxido de aleno intermedio, compuesto que ha sido estudiado también por RMN (41). La especificidad de sustrato de este enzima también ha sido estudiado y se vió que los siguientes hidroperóxidos de ácidos grasos servían como sustratos y eran convertidos en óxidos de aleno: hidroperóxidos en las posiciones 9 y 13 derivados del ácido linoleico, 13-hidroperóxido del ácido $\alpha$-linolénico, 9-hidroperóxido del ácido $\gamma$-linolénico, 15 hidroperóxido del ácido bis-homo- $\alpha$-linolénico, 15-hidroperóxido del ácido araquidónico y 15-hidroperóxido del ácido eicosapentanoico (42).

Los óxidos de aleno son muy reactivos y sufren una hidrólisis rápida en medio acuoso dando los correspondientes cetoles. Cuando se parte del ácido 12,13(S)-epoxi-9(Z),11-octadecadienoico el compuesto mayoritario resulta ser el $\alpha$-cetol derivado (ácido 12-oxo-13-hidroxi-9(Z)-octadecenoico). Análisis de la estereoquímica de este $\alpha$-cetol mostró que el mismo tenía una configuración $R$ mayoritaria en el C-13 (39). Por tanto la formación de los $\alpha$-cetoles a partir de los óxidos de aleno aparentemente ocurría mayoritariamente por una sustitución nucleofílica $S_{N} 2$ en el carbono saturado del epóxido (lo que provoca una inversión de la configuración). La pequeña racemización observada debe ser debida a una reacción de tipo $S_{N} 1$ en la que toma parte un ión divalente de tipo oxipentadienilo.

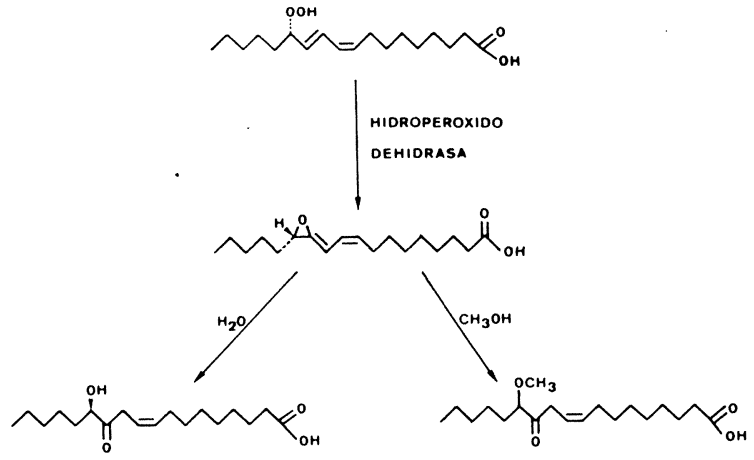

Figura 4

Formación del óxido de aleno a partir del correspondiente hidroperóxido por acción de la hidroperóxido dehidrasa y posterior reacción de este óxido de aleno con metanol y agua, respectivamente.

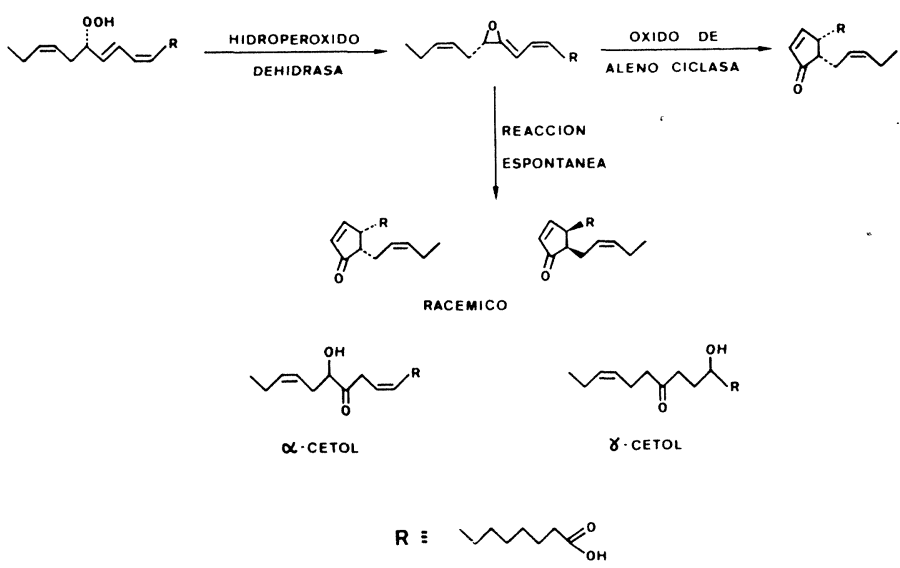

Figura 5

Transformación del 13-hidroperóxido del ácido linolénico en ácido $9(S), 13(S)$-12-oxofitodienoico por acción de la óxido de aleno ciclasa y en $\alpha$ - y $\gamma$-cetoles por hidrólisis del óxido de aleno intermediario. 


\section{OXIDO DE ALENO CICLASA}

Cuando los óxidos de aleno son estabilizados con albúminas de suero (43) la vida media de estos compuestos sube de 30 segundos a 15 minutos. Esta estabilización es probablemente debida al enlace de los óxidos de aleno a los bolsillos hidrofóbicos de la molécula de albúmina lo que resulta en una protección frente a la hidrólisis que provoca la fase acuosa. En este caso la reacción evoluciona hacia ácidos ciclopentenoctanoicos del tipo de la Figura 5. Compuestos análogos a estos habían sido encontrados con anterioridad por Zimmerman y Feng en 1978 (44) cuando observaron la conversión del ácido $\alpha$-linolénico en ácido 12-oxo-10,15(Z)-fitodienoico. El paso inicial de la conversión consistía en la formación del 13-hidroperóxido del ácido linolénico. Un enzima denominado hidroperóxido ciclasa fue entonces propuesto para explicar la conversión en la ciclopentanona. Como se verá posteriormente el ácido oxofitodienoico es el precursor del ácido jasmónico (45), un compuesto que ha sido aislado de numerosas plantas y que tiene efectos reguladores del crecimiento (46).

Los óxidos de aleno han sido propuestos como intermediarios en la formación del ácido 12-oxofitodienoico en plantas $(44,47,48)$ a causa de la bien establecida conversión química de los óxidos de aleno en ciclopentanonas $(49,50)$ y porque la formación del ácido 12-oxofitodienoico es acompañada de la formación de $\alpha$-cetoles. Aunque esta reacción puede ser también no enzimática, como ocurre con la formación del ácido 12-oxofitodienoico que se obtiene racémico usando un extracto de lino obtenido por precipitación con acetona (47), muy recientemente ha sido aislado y caracterizado un enzima responsable de la conversión del óxido de aleno en ácido 12-oxofitodienoico. Este enzima ha sido denominado óxido de aleno ciclasa $(51,52)$.

La existencia de este enzima fue sugerida cuando una preparación de homogeneizado de maíz, a diferencia del lino catalizó la formación de un ácido 12 oxofitodienoico que no era racémico sino una mezcla de los enantiómeros $9(\mathrm{~S}), 13(\mathrm{~S})$ y $9(\mathrm{R}), 13(\mathrm{R})$ en proporción 82:18 (53). Además, el hecho de que ácido jasmónico ópticamente activo sea sintetizado a partir del ácido 12-oxofitodienoico por plantas (36) sugería que al ácido 12-oxofitodienoico sintetizado en la planta intacta debería ser el enantiómero $9(S), 13(S)$ y no el racémico.

Hamberg encontró que la fracción soluble de un extracto de maíz contenía una proteína de peso molecular 45000 y que tenía actividad de óxido de aleno ciclasa (52). Se comprobó que el sustrato de este enzima era el óxido de aleno y no el 13-hidroperóxido del linoleico o el cetol derivado de dicho óxido de aleno. El producto formado con la misma fue exclusivamente el enantiómetro $9(\mathrm{~S}), 13(\mathrm{~S})$ del ácido 12-oxofitodienoico, aunque una cierta proporción de racémico, que varió con las condiciones, también se formaba como consecuencia de reacciones no enzimáticas. La óxido de aleno ciclasa exhibe especificidad de sustrato hacia el óxido de aleno derivado del 13-hidroperóxido del ácido linolénico no sirviendo el derivado del 9-hidroperóxido que produjo una ciclopentanona racémica (52).

Esta óxido de aleno ciclasa ha sido encontrada en distintas plantas, siendo las más ricas en ella la patata, las hojas de espinacas y la col blanca (52).

\section{ACIDO 12-OXOFITODIENOICO REDUCTASA}

La conversión del ácido 12-oxofitodienoico en ácido jasmónico transcurre en varios pasos (54), siendo el primero de ellos la reducción del doble enlace del anillo de ciclopentenona (Figura 6). Este paso es producido por la ácido 12-oxofitodienoico reductasa (55). Este enzima, que necesita la presencia de un donador de átomos de hidrógeno de los que el NADPH funciona mucho mejor que $\mathrm{NADH}$, tiene un $\mathrm{pH}$ óptimo de 6.8-9. El enzima aislado del maíz tiene un peso molecular de aproximadamente 54000. Su baja actividad en comparación con la lipoxigenasa o la hidroperóxido dehidrasa sugieren que este enzima puede ser el paso limitante que determina la cantidad de
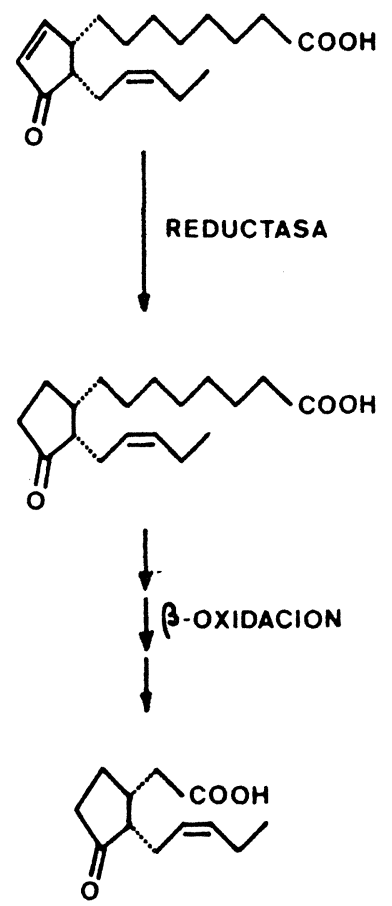

ACIDO

JASMONICO

Figura 6

Formación del ácido jasmónico a partir del ácido 12-oxofitodienoico. 
ácido 12-oxofitodienoico que es convertido a jasmónico (55).

El ácido 12-oxofitodienoico producido por la ácido 12-oxofitodienoico reductasa sufre entonces tres ciclos de B-oxidación. El producto final, ácido jasmónico, retiene la misma configuración cis de las cadenas laterales.

La formación de este ácido jasmónico se ha observado en diversas especies vegetales entre las que se pueden señalar haba, maíz, lino, girasol y trigo entre otras, por lo que parece ser una ruta metabólica general en plantas (31).

\section{OTROS PRODUCTOS DEL METABOLISMO DE LOS HIDROPEROXIDOS}

Una última reacción de transformación de los hidroperóxidos, de la que es responsable un sistema enzimático que se ha encontrado exclusivamente en la patata es la formación de los diviniléteres de la Figura 7 que son producidos a partir del 9-hidroperóxido de los ácidos linoleico y linolénico, pero no cuando se parte de los 13-hidroperóxidos o de los ácidos sin oxidar (56). También es importante el pH. $\mathrm{Su}$ formación de produce exclusivamente a $\mathrm{pH}$ alcalinos. El mecanismo de la reacción es desconocido. Estudios con hidroperóxidos marcados con ${ }^{18} \mathrm{O}$ en los oxígenos del grupo hidroperóxido producen éteres en los que el oxígeno etéreo no está marcado. Estos éteres representan la fracción lipídica principal en homogeneizados de patata a $\mathrm{pH}>6.5$. Esto éteres vinílicos se pueden degradar posteriormente a compuestos volátiles dando los correspondientes nonenales.

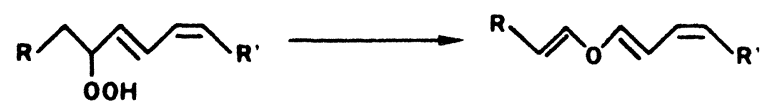

Figura 7

Transformación del 9-hidroperóxido de los ácidos linoleico $y$ linolénico en diviniléteres producida en la patata.

\section{MECANISMOS DE CONTROL DE LA RUTA}

La degradación de los ácidos grasos poliinsaturados tiene lugar por acción de los diversos enzimas que se han venido describiendo en este trabajo. El intermedio central en este proceso es el hidroperóxido del ácido graso, el cual puede ser dirigido a alguna de las diferentes rutas que se han descrito. La formación de los diversos hidroperóxidos y la posterior transformación de los mismos va a venir determinada en mayor o menor medida por la especificidad de los isoenzimas de la lipoxigenasa y los otros enzimas que contenga la planta. Esto hace, por ejemplo, que la concentración y variedad de volátiles sea tan diferente de una planta a otra.
Así en hojas de té, donde esta ruta ha sido muy estudiada, se ha encontrado que la lipoxigenasa produce mayoritariamente el 13-hidroperóxido y la hidroperóxido liasa transforma este 13-hidroperóxido (57). Esto produce los hexanales y hexenales encontrados en hojas maceradas que son las que le dan el olor herbáceo. Al igual que en hojas de té, los principales productos de rotura en el tomate son compuestos $\mathrm{C}_{6}$. Al (Z)-3-hexenal se atribuye principalmente el aroma del tomate fresco (58) y es producido tras la oxidación del ácido linoleico que sigue a la maceración de los tomates. Por su parte las notas frescas del pepino o las violetas son debidas a compuestos volátiles $C_{9}$ siendo el 2,6-nonadienal el responsable principal.

Aparte de esta especificidad de los enzimas, la proporción final de volátiles va a depender de inhibidores naturales que se han encontrado para los diversos enzimas descritos, principalmente para los más estudiados lipoxigenasa e hidroperóxido liasa, y que podrían actuar como un cierto mecanismo de control en la ruta. La formación de hexanal a partir de ácido linoleico por la secuencia lipoxigenasa-liasa en inhibida tanto por el ácido linolénico como por el 13-hidroperóxido del mismo ácido (59). Por el contrario la formación del (Z)-3-hexenal no es inhibida por el ácido linoleico o su hidroperóxido. Esto explica que la proporción (Z)-3-hexenal/hexanal en hojas de té sea mucho mayor que la relación ácido linoleico/linolénico. Otros inhibidores son el ácido palmítico, que inhibe la formación de hexanales y hexenales (59), y los monogalactosildiglicéridos, que retardan la formación de hexanal (60). También se ha visto que en semillas de pepino, en alfalfa (61) y en hojas de té la acción de la hidroperóxido liasa sobre el 13-hidroperóxido del ácido linoleico fue retardada por el 13-hidroperóxido del ácido linolénico aunque no por el ácido linolénico (50). Otros inhibidores de la producción de aldehídos $C_{6}$ han sido encontrados en semillas de arroz, cotiledones de judías y semillas de té (62).

Por último, otro factor que también influye es la temperatura. Así por ejemplo, en hojas de té, que es tal vez la planta donde más se ha estudiado esta ruta, se ha visto que la producción de los $\mathrm{C}_{6}$ es estacional, debido a los cambios que sufren las actividades de la lipoxigenasa y la hidroperóxido liasa a lo largo del año. Estos cambios están a su vez estrechamente relacionados con las temperaturas y las horas de sol. Cuando las temperaturas están por debajo de $10^{\circ} \mathrm{C}$ la producción de hexanales decae hasta casi desaparecer mientras que entre julio y agosto alcanza su máxima actividad cuando las horas de sol y las temperaturas son máximas. El hecho de que durante todo el año haya actividad liasa mientras que actividad lipoxigenasa no y que esta última tenga su máximo entre julio y agosto indica que la etapa determinante en estos cambios estacionales en la producción de $\mathrm{C}_{6}$ es la lipoxigenasa (57). 
Todos estos datos parecen indicar que las alteraciones bioquímicas de los lípidos en alimentos vegetales empiezan a ser comprendidas y por tanto pueden empezar a ser controladas. Sin embargo, aún quedan muchos puntos oscuros en los que profundizar como puede ser la función fisiológica de los productos originados en la cascada. En el futuro, los avances sobre los análogos a las prostaglandinas y leucotrienos en plantas, los que ya comienzan a ser conocidos como octadecanoides, prometen ser espectaculares.

\section{AGRADECIMIENTO}

Este trabajo ha sido financiado en parte por la Comisión Interministerial de Ciencia y Tecnología (Proyecto ALI 88-0169) y la Junta de Andalucía (Proyecto 2075). Los autores desean agradecer al Prof. Eduardo Vioque sus valiosas discusiones durante la realización del mismo.

\section{BIBLIOGRAFIA}

1. Zamora, R.; Hidalgo, F.J., y Alaiz, M.-"Alteraciones bioquímicas de los lípidos en los alimentos vegetales. I. Formación de los hidroperóxidos lipidicos".-Grasas y Aceites 42 (1991) 155-162.

2. Gardner, H.W.-"Reactions of hydroperoxides.-Products of high molecular weight", en "Autoxidation of Unsaturated Lipids", pp. 51-93.-Chan, H.W.S. (Ed.), Academic Press, London, 1987.

3. Grosch, W.-“Reactions of hydroperoxides.-Products of low molecular weight", en "Autoxidation of Unsaturated Lipids".-pp. 95139.-Chan, H.W.S. (Ed.), Academic Press, London, 1987.

4. Frankel, E.N.-"Secondary products of lipid oxidation".-Chem. Phys. Lipids 44 (1987) 73-85.

5. Vioque, E., y Holman, R.T.-"Characterization of the ketodienes formed in the oxidation of linoleate by lipoxidase".-Arch. Biochem. Biophys. 99 (1962) 522-528.

6. Axelrod, B.-"Lipoxygenases" en "Food Related Enzymes".-pp. 324-348.-Whitaker, J.R. (Ed.), Adv. Chem. Ser. 136, A.C.S. Washington, D.C., 1974.

7. Sessa, D.J.; Gardner, H.W.; Kleiman, R., y Weisleder, D.-"Oxygenated fatty acids constituents of soybean phosphatidylcholines".-Lipids 12 (1977) 613-619.

8. Moll, C.; Biermann, V., y Grosch, W.-"Occurrence and formation of bitter-tasting trihydroxy fatty acids in soybeans".J. Agric. Food Chem. 27 (1979) 239-243.

9. Esterbauer, H., y Schauenstein, E.-"Isomeric trihydroxy-octadecenoic acids in beer: Evidence for their presence and quantitative determination".-Z. Lebensm. Unters.-Forsch. 164 (1977) 255-259.

10. Fioriti, J.A.; Bentz, A.P., y Sims, R.J.-"The reaction of picric acid with epoxides. I. A colorimetric method".J. Am. Oil Chemists' Soc. 43 (1966) 37-41.

11. Garssen, G.J.; Vliegenthart, J.F.G., y Boldingh, J.-"The origin and structures of dimeric fatty acids from the anaerobic reaction between soyabean lipoxygenase, linoleic acid, and its hydroperoxide".-Biochem. J. 130 (1972) 435-442.

12. De Groot, J.J.M.C.; Veldink, G.A.; Vliegenthart, J.F.G.; Boldingh, J.; Wever, R., y van Gelder, B.F.-"Demonstration by EPR spectroscopy of the functional role of iron in soybean lipoxygenase1".-Biochim. Biophys. Acta 377 (1975) 71-79.
13. Verhagen, J.; Bouman, A.A.; Vliegenthart, J.F.G., y Boldingh, J.-Conversion of 9-D- and 13-L-hydroperoxylinoleic acids by soybean lipoxygenase-1 under anaerobic conditions".-Biochim. Biophys. Acta 468 (1977) 114-120.

14. Bohn, R.M., y Hass, L.W. (1928). Citado en "Chemistry and Methods of Enzymes".-3rd Ed.-pp. 311.-Summer, J.B., y Somers, G.F., (Eds.), Academic Press, New York, 1985.

15. Veldink, G.A.; Vliegenthart, J.F.G., y Boldingh, J.-“Plant lipoxygenases".-Prog. Chem. Fats Others Lipids 15 (1977) 131 136.

16. Imamura, M., y Shimizu, S.-"Metabolism of chlorophyll in higher plants. IV. Relationship between fatty acid oxidation and chorophyll bleaching in plant extracts".-Plant Cell Physiol. 15 (1974) 187-190.

17. Grosch, W.-'Linoleic and linolenic acids as substrate for enzymatic formation of volatile carbonyl compounds in pea".-Z Lebensm. Unters. Forsch. 137 (1968) 216-223.

18. Grosch, W., y Schwencke, D.-“Soybean lipoxygenase: volatile aldehydes and alcohols from linoleic acid".-Lebensm.-Wiss. Technol. 2 (1969) 109-112.

19. Fischer, K.H., y Grosch, W.-"Cooxidation of linoleic acid to volatile compounds by lipoxygenase isoenzymes from soya beans". $-Z$. Lebensm. Unters. Forsch. 165 (1977) 137-139.

20. Grosch, W., y Laskawy, G.-“Differences in the amount and range of volatile carbonyl compounds formed by lipoxygenase isoenzymes from soybeans".-J. Agric. Food Chem. 23 (1975) 791-794.

21. Grosch, W.-"Breakdown of linoleic acid hydroperoxide. Formation of volatile carbonyl compounds".-Z. Lebensm. Unters. Forsch. 160 (1976) 371-375.

22. Vliegenthart, J.F.G.; Veldink, G.A., y Boldingh, J.--"Recent progress in the study on the mechanism of action of soybean lipoxygenase".-J. Agric. Food Chem. 27 (1979) 623-626.

23. Hatanaka, A.; Kajiwara, J.; Sekiya, J., e Inouye, S.-"Solubilization and properties of the enzyme-cleaving 13-L-hydroperoxylinoleic acid in tea leaves".-Phytochemistry 21 (1982) 13-17.

24. Wardale, D.A.; Lambert, E.A., y Galliard, T.-"Localitation of fatty acid hydroperoxide cleavage activity in membranes of cucumber fruit".-Phytochemistry 17 (1978) 205-212.

25. Galliard, T.; Phillips, D.R., y Reynolds, J.-“The formation of cis3-nonenal, trans-2-nonenal and hexanal from linoleic acid hydroperoxide isomers by a hydroperoxide cleavage enzyme system in cucumber (Cucumis sativus) fruits".-Biochim. Biophys. Acta 441 (1976) 181-192.

26. Kim, I.S., y Grosch, W.-"Partial purification and properties of a hydroperoxide lyase from fruits of pear".-J. Agric. Food Chem. 29 (1981) 1220-1225.

27. Wurzenberger, M., y Grosch, W.-"The formation of 1-octen-3ol from the 10-hydroperoxide isomer of linoleic acid by a hydroperoxide lyase in mushrooms (Psalliota bispora)".-Biochim. Biophys. Acta 794 (1984) 25-30.

28. Gardner, H.W.-"Flavor and bitter tastes from oxidation of lipids by enzymes" en "Flavor Chemistry of Fats and Oils".-pp. 189. 206.-Min, D.B., y Smouse, T.H. (Eds.), American Oil Chemists Society, 1985.

29. Borgeat, P.; Hamberg, M., y Samuelsson, B.-"Transformation of arachidonic acid and homo- $\gamma$-linolenic acid by rabbit polymorphonuclear leukocytes: monohydroxy acids from novel lipoxygenases".-J. Biol. Chem. 251 (1976) 7816-7820.

30. Whitaker, J.R.-"Mechanisms of oxidoreductases important in food component modification" en "Chemical Changes in Food During Processing".-pp. 121-176.-Richardson, T., y Finley, J.M. (Eds.), AVI Publishing Company, Inc. Westport, Connecticut, 1985.

31. Vick, B.A., y Zimmerman, D.C.-"Oxidative systems for modification of fatty acids: the lipoxygenase pathway" en "The Biochemistry of Plants. A Comprehensive Treatise. Lipids: structure and function".-Vol. 9, pp. 53-90.-P.K. Stumpf, y Conn, E.E. (Eds.), Academic Press, Inc. New York, 1987. 
32. Zimmerman, D.C.-"A new product of linoleic acid oxidation by a flaxseed enzyme".-Biochim. Biophys. Res. Commun. 23 (1966) 398-402.

33. Yabuuchi, S., y Amaha, M.-“Partial purification and characterization of the linoleate hydroperoxide isomerase from grains of Hordeum distichum".-Phytochemistry 15 (1976) 387-390.

34. Lulai, E.C.; Backer, C.W., y Zimmerman, D.C.-"Metabolism of linoleic acid by barley lipoxygenase and hydroperoxide isomerase".-Plant Physiol. 68 (1981) 950-955.

35. Gardner, H.W.-"Sequential enzymes of linoleic acid oxidation in corn germ: lipoxygenase and linoleate hydroperoxide isomerase".-J. Lipid Res. 11 (1970) 311-321.

36. Vick, B.A., y Zimmerman, D.C.-"Lipoxygenase, hydroperoxide isomerase, and hydroperoxide cyclase in young cotton seedlings".-Plant Physiol. 67 (1981) 92-97.

37. Grossman, S.; Bergman, M., y Sofer, Y.-"Purification and partial characterization of eggplant linoleate hydroperoxide isomerase".-Biochim. Biophys. Acta 752 (1983) 65-72.

38. Vick, B.A., y Zimmerman, D.C.-"Distribution of a fatty acid cyclase enzyme system in plants".-Plant Physiol. 64 (1979) 203-205.

39. Hamberg, M.-"Mechanism of corn hydroperoxide isomerase: detection of 12,13(S)-oxido-9(Z),11-octadecadienoic acid".Biochim. Biophys. Acta 920 (1987) 76-84.

40. Vick, B.A., y Zimmerman, D.C.-“Pathway of fatty acid hydroperoxide metabolism in spinach leaf chloroplasts".-Plant Physiol. 85 (1987) 1073-1078.

41. Brash, A.R.; Baertschi, S.W.; Ingram, C.D., y Harris, T.M.-"Isolation and characterization of natural allene oxides: unstable intermediates in the metabolism of lipid hydroperoxides".-Proc. Natl. Acad. Sci. USA 85 (1988) 33823386.

42. Hamberg, M.-"Fatty acids allene oxides".-J. Am. Oil Chemists' Soc. 66 (1989) 1445-1449.

43. Hamberg, M., y Hughes, M.-"Fatty acid allene oxides. III. Albumin-induced cyclization of $12,13(\mathrm{~S})$-epoxy-9(Z),11-octadecadienoic acid".-Lipids 23 (1988) 469-475.

44. Zimmerman, D.C., y Feng, P.-“Characterization of a prostaglandin like metabolite of linolenic acid produced by a flaxseed extract".-Lipids 13 (1978) 313-316.

45. Vick, B.A., y Zimmerman, D.C.-"The biosynthesis of jasmonic acid: a physiological role for plant lipoxygenase".-Biochem. Biophys. Res. Commun. 111 (1983) 470-477.

46. Sembdner, G., y Klose, C.-"(-)-Jasmonic acid a new phytohormone?".-Biol. Rundsch. 23 (1985) 29-40.

47. Baertschi, S.W.; Ingram, C.D.; Harris, T.M., y Brash, A.R.-"Absolute configuration of cis-12-oxophytodienoic acid of flaxseed: implications for the mechanism of biosynthesis from the 13(S)-hydroperoxide of linolenic acid".-Biochemistry 27 (1988) 18-24.

48. Crombie, L., y Morgan, D.O.--Formation of acyclic $\alpha-y \quad \gamma$-ketoles and 12-oxophytodienoic acid from linolenic acid 13-hydroperoxide by a flax enzyme preparation. Evidence for a single enzyme leading to a common allene epoxide intermediate".-J. Chem. Soc. Chem. Commun. (1988) 558-560.
49. Grimaldi, J., y Bertrand, M.-"Epoxidation of vinylallenes. Formation of conjugated cyclopentenones".-Tetrahedron Lett. 38 (1969) 3269-3272.

50. Malacria, M., y Roumestant, M.L.-"Vinylallenes. VI. Synthesis of ketones of the jasmonic series".-Tetrahedron Lett. 33 (1977) 2813-2817.

51. Hamberg, M.-"Biosynthesis of 12-oxo-10,15(Z)-phytodienoic acid identification of an allene oxide cyclase".-Biochem. Biophys. Res. Commun. 156 (1988) 543-550.

52. Hamberg, M., y Fahlstadius, P.-"Allene oxide cyclase: a new enzyme in plant lipid metabolism".-Arch. Biochem. Biophys. 276 (1990) 518-526.

53. Hamberg, M.; Miersch, O., y Sembdner, G.-"Absolute configuration of 12-oxo-10,15(Z)-phytodienoic acid".-Lipids 23 (1988) 521-524.

54. Vick, B.A., y Zimmerman, D.C.-"Biosynthesis of Jasmonic Acid by Several Plant Species".-Plant Physiol. 75 (1984) 458-461.

55. Vick, B.A., y Zimmerman, D.C.-"Characterization of 12-oxo-phytodienoic acid reductase in corn. The jasmonic acid pathway".-Plant Physiol. 80 (1986) 202-205.

56. Galliard, T., y Matthew, J.A.-"Enzynic reactions of fatty acid hydroperoxides in extracts of potato tuber. I. Comparison 9-Dand 13-L-hydroperoxy-octadecadienoic acid as substrates for the formation of a divinyl ether derivative".-Biochim. Biophys. Acta 398 (1975) 1-9.

57. Hatanaka, A.; Kajiwara, T., y Sekiya, J.-“Biosynthetic pathway for $\mathrm{C}_{6}$-aldehydes formation from linoleic acid in green leaves".Chem. Phys. Lipids 44 (1987) 341-361.

58. Kazeniac, S.J., y Hall, R.M.-"Flavor chemistry of tomato volatiles".-J. Food Sci. 35 (1970) 519-530.

59. Hatanaka, A.; Sekiya, J., y Kajiwara, T.-"Linolenic acid and its 13-hydroperoxide inhibit hexanal formation from linoleic acid in plant tissues".-J. Agric. Food Chem. 31 (1983) 176-178.

60. Sekiya, J.; Kajiwara, T.; Imoto, M.; Inouye, S., y Hatanaka A.-"Effect of lipolytic acid hydrolase on the activity for six carbon aldehyde formation in the tea chloroplasts".-J. Agric. Food Chem. 30 (1982) 183-185.

61. Sekiya, J.; Kajiwara, T., y Hatanaka, A.-“Volatile $C_{6}$-aldehyde formation via hydroperoxides from $\mathrm{C}_{18}$-unsaturated fatty acids in etiolated alfalfa and cucumber seedlings".-Agric. Biol. Chem. 43 (1979) 969-980.

62. Hatanaka, A.; Sekiya, J.; Kajiwara, T., y Munechika, K.-“Natura

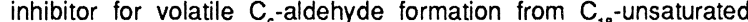
fatty acids".-Agric. Biol. Chem. 46 (1982) 2705-2710. 